\title{
Probiotics, Prebiotics and Children's Intestinal Health
}

\author{
Ruokun $\mathrm{Yi}^{1,2, \mathrm{a}}$ and Xin Zhao ${ }^{1,2, \mathrm{~b}^{*}}$ \\ ${ }^{1}$ Chongqing Collaborative Innovation Center for Functional Food \\ ${ }^{2}$ School of Biological and Chemical Engineering, Chongqing University of Education, Chongqing \\ 400067, China \\ ayirk@cque.edu.cn, bzhaoxin@cque.edu.cn \\ ${ }^{*}$ Corresponding author
}

Keywords: Probiotics; Prebiotics; Children; Intestinal health

\begin{abstract}
A lot of researches have verified the protection effect of probiotics, prebiotics and their mixed preparation for the intestinal health of children. Probiotics promote absorption and protect alimentary canal of organism; prebiotics stimulate the growth of useful flora and have maintaining effect for the balance of intestinal flora. Nowadays, probiotics and prebiotics have become a functional substance to promote intestinal health and remedy intestines disease for children.

The inner wall of intestines is the largest area which human body connects external environment. The research shows that fetus grows and develops in the aseptic environment of maternal womb. After birth, the infant will contact the maternalmicrobiota. Consequently, the newborn child will obtain intestinal flora within several hours after birth. Different infants have different intestinal flora when birth. In the second day after birth, bacteroides bacteria, bifidobacterium and clostridium have appeared in a half of infants. From the fourth to fifth day, the quantity of enteric bacilli is decreased gradually. From the sixth to eighth day, the flora taking bifidobacterium as leading role has been established. But in the last stage of suckling period, it will be translated to the system similar to intestinal flora of adult human. All kinds of bacteria carried by human body have an inseparable relationship with the nutrition, digestive absorption, immunity and other functions of body itself [1].
\end{abstract}

\section{Probiotics}

In 1899, Henry Tissier from Paris Children's Hospital of France separated the first bifidobacterium from the feces of infant through health breast nursing first. He discovered that bifidobacterium was related to the frequency of diarrhea and the nutrition of infant. It arose the research enthusiasm of whole world for the correlation between probiotics and intestinal health. Nowadays, probiotics has become a functional substance to promote intestinal health and remedy intestines disease for children even the adult. According to the definition proposed by the expert group of United Nations Food and Agriculture Organization and World Health Organization in 2001, probiotics is a microorganism given a certain quantity and beneficial to the health of its host. The protective effect of probiotics for human organism has the mechanism as follows.

i. Promoting absorption, nourishing organism: enzymes generated by probiotics can decompose the substances which the human cannot digest or has difficulty in digestion. For instance, bacteroides has a series of enzymes to digest polysaccharide. It can decompose the polysaccharide which human body cannot digest like cellulose and hemicellulose etc. in plant so as to provide energy for human body. The intermediate metabolite of probiotics like short-chain fatty acid can make the growth of intestinal epithelial cell more active and to generate more intestinal crypt whose increase can promote the absorption of small intestine. In addition, probiotics also can promote the absorption of nutrient in organism. For instance, bifidobacterium can compound B-Vitamins. Moreover, it also can inhibit the aneurinibacillus which decomposes Vitamin B1.

ii. Protecting alimentary canal of organism: probiotics can form the biological barrier in intestinal canal. In addition, it also can protect the alimentary canal of human body through reducing environmental $\mathrm{pH}$, oxygen concentration, redox potential and accelerating the proliferation and metabolism of intestinal epithelial cellas well as increasing the mucus secretion of intestinal 
canal etc. For instance, aerobic bacterium, bacillus subtilis consumes free oxygen and reduce the oxygen concentration of intestinal canal and redox potential so as to create a better living environment for the anaerobic probiotics like bifidobacterium etc. after it entering the intestinal canal of human body.

Meanwhile, probiotics can secrete the substances to resist pernicious bacteria. For instance, bifidobacterium can generate the antibiotic substance, bifidin. It can inhibit saprophytic bacteria to reduce benzazole and nitrosamine etc. significantly. Moreover, probiotics often is applied in the clinical treatment or adjuvant therapy of gastrointestinal tract.

Some probiotics also can be used in the immune system of human body directly, induce intestinal immunity and simulate immune organs like thymus gland etc. so as to strengthen the activity of macrophage. The specific immunocompetence can be brought into play through promoting the reactivity of lymphocyte for antigen to strengthen the immune function of organism $[2,3,4,5]$.

Thereinto, the relationship between intestinal health of children and probiotics also has been clarified by a large quantity of clinical researches (like L.rhamnosus GG, L.casei Shirota, L.reuterii, B.bifidum etc) [6]. Diarrhea is one of reasons resulted in the disease and death of children under 5 years old. Diarrhea is ranked the second in the most common diseases which results in the death of children under 5 years old in the world, second only to pneumonia [7].

Rotavirus is one of primary pathogens which results in infantile diarrhea. The infants died from rotavirus infection are 900000 approximately in the world every year. In China, the infants between 0 to 2 years old are 40 million approximately (including the newborn). There are about 10 million infants suffering from rotavirus infective gastroenteritis every year, which accounts for $1 / 4$ of the infants. 48.4\%-77.1\% diarrhea of infants under 5 years old is caused by rotavirus infection [8]. This rotavirus mainly infects intestinal epithelial cell to cause cellular damage and diarrhea. Most researches uphold that the change of intestinal flora is caused by rotavirus enteritis. It is reflected by the decrease of health anaerobic bacteria like bifidobacterium and lactobacillus etc. to further increase the infection opportunity of pathogenic bacteria [9].

The clinical observation shows that the infants with intestinal flora disturbance in the treatment group with probiotics and their defecating frequency are obvious better than the control group of infants in the later period of rotavirus disease after the probiotics is used. Moreover, the probability to induce bacterial enteritis disease is obvious lower than the one in control group. This has verified that probiotics can speed up the normal function of intestinal mucosal barrier, keep the integrity of enterocyte, adjust the growth and reproduction of gastrointestinal bacteria through repairing the intestinal mucosal injury resulted from virus infection to relieve intestinal flora disturbance, reduce the diarrhea frequency and the risk of secondary bacterial infection [10].

In addition, a research has found that the diarrhea lasting for above 3 days can be reduced when using probiotics. This research compares 731 hospitalized children and outpatient children suffered from acute infective diarrhea at 1 to 4 years old when using probiotics(including lactobacillus GG, saccharomyces boulardii and lactobacillus reuteri) and placebo through a randomized controlled experiment. There are also some randomized controlled experiments verifying the incident rate of ADD (antibiotic-associated diarrhea)1015infants in experimental group is reduced to $8.9 \%$ from $37.5 \%$ after using probiotics [11].

\section{Prebiotics}

Prebiotics was defined as follows: "prebiotics is a specific ingredient which can be fermented selectively and change the composition and active of flora, and beneficial to the health of host." According to the definition, prebiotics should meet the following standards:

i. Harmless to human body;

ii. Not be digested by digestive enzyme in gastrointestinal system;

iii. Stimulating the growth of useful flora and having maintaining effect for the balance of intestinal flora;

iv. Its metabolite (like short-chain fatty acid) has a certain functional effect. 
Basically, prebiotics is carbohydrate. But this definition doesn't exclude non-carbohydrate substances used as prebiotics. In theory, allbacteria or the active substance which can decrease the current harmful bacteriaand benefit to the health can be called prebiotics. It mainly includes all kinds of oligosaccharide or known as oligose. To summarize, it is a functional oligose. The indicators to assess the effect of prebiotics are as follows: multiplication effect for beneficial bacteria (like bifidobacterium and lactobacillus); inhibition for harmful bacteria (like clostridium) and non-multiplication effect forpotential pathogenic bacteria (like escherichia coli, enterococcus and bacteroidetes etc.); acid and gas production output after metabolized by intestinal flora [12]. The most common oligose which has realized the industrial scale production in China mainly includes fructo-oligose, galactooligosaccharide and isomalto-oligosaccharide.

It is found that the bifidobacterium in the intestinal canal of breast-feeding infant accounts for about $99 \%$ of anaerobion while it only accounts for about $90 \%$ for the artificial-feeding infant when through detecting and analyzing the intestinal flora of infant [13]. The reason is that there is a substance, called N-acetyl-D-glucose in human milk, whose content is the most in human colostrum. This substance can promote the reproduction and multiplication of bifidobacterium. This substance also can be called bifidus factor. Consequently, bifidus factor is added in theinfant formula milk powder, which has become one of most effective measures to maintain the intestinal health of infant.

Moro et al. add $4 \mathrm{~g} / \mathrm{L}$ and $8 \mathrm{~g} / \mathrm{L}$ oligose mixture in infant formula milk powder, among which $90 \%$ is galacto-oligosaccharide and 10\%, fructo-oligose. After feed for 28 days continuously, the bifidobacterium and the lactobacillus in intestinal canal of infants in experimental group aremultiplied obviously. The $\mathrm{pH}$ of faeces is reduced. The moisture and the frequency of faeces are increased against control group. In addition, the effect of breastfeeding when the addition amount is $8 \mathrm{~g} / \mathrm{L}$. Consequently, prebiotics also becomes an important factor to strengthen the intestinal health of infant [14].

There are some problems difficult to be solved for the exogenous supplement of viable bacteria, so scientists have turned their attention to the research on preparation of bifidus factor. This research is carried out in Japan more early. It is found through the proliferationexperiment of bifidobacterium in vitro that the substance promoting the proliferation of bifidobacterium can be separated from not only the human milk but also the natural substances. But the proliferation factor which can be made as preparation product is little. This is because that bifidobacterium mainly is located in the end part of small intestine and colon as the proliferation factor. It must not be broken, digested and absorbed when passing the front end of small intestine. For instance, although glucose makes bifidobacterium multiplied in vitro, it has been absorbed at the front end of small intestine. It can't reach colon. Therefore, it is not suitable to produce the bifidus proliferation preparation. In this way, some oligose and polysaccharide which cannot be absorbed by human body are screened out to reach colon with the original form and be used by intestinal bacteria.

However, many of them are eliminated because they can be made used by the harmful bacteria. The retained one is not absorbed by human body and not made used by the harmful bacteria, either, and as the substance only made used by bifidobacterium to be developed as bifidus factor. The developed one includes catabolic lactose oligosaccharides, lactuloseoligosaccharides, xylooligosaccharide and soybean oligosaccharides etc $[15,16]$.

At present, it is said that the best probiotics carrier is fermented dairy product for children. The main reason is that the fermented dairy product has a good health effect for each function of body including growth and development etc. Secondly, the producing technology of this product can keep the most viable counts of zymophyte. In China, Ministry of Health also has implemented Administrative Measures for the Safety Review of New Food Raw Materials and approved part of bacteria can be used as the new food raw materials of infant food (like Lactobacillus rhamnosus, Bifidobacterium lactis, Lactobacillus acidophilus etc).

The appearance of prebiotics has improved the defects that living bacteria preparation is easy to be inactivated by the digestive enzyme of host and excreted from body etc. On the one hand, it can promote the survival and constant value of probiotics. On the other hand, it can promote the 
multiplication of native beneficial bacteria. It has become an important substance for the new materials and nutrient supplements of infant food as well as the clinical adjuvant therapy of gastrointestinal disorders etc.

A large quantity of researches have verified the protection effect of probiotics, prebiotics and their mixed preparation for the intestinal health of children. However, its use ratio by human body still is a problem difficult to be solved at present. For instance, the living bacteria preparation is easy to be inactivated in the process of production, transportation and storage, which has reduced its biological activity significantly. Besides, when entering the intestinal canal, they grows slowly and difficult to dominate in the microbial environment of intestinal canal. It is worth to make a further study for probiotics and prebiotics.

\section{Acknowledgements}

The present research was supported by the Program for Innovation Team Building at Institutions of Higher Education in Chongqing (grant no. CXTDX201601040) and the Construction Program of Chongqing Engineering Research Center (grant no. cstc2015yfpt_gcjsyjzx0027).

\section{References}

[1] M.H. Floch and A.S. Kim: Probiotics: A Clinical Guide(SLACK Inc., NJ, USA, 2010).

[2] M.H. Floch: Journal of Clinical Gastroenterol. Vol.45(2011), p.108.

[3] M.H. Floch, W.A. Walker, S Guandalini et al: Journal of Clinical Gastroenterol. Vol.42 (2008), p.104.

[4] P. Marteu: Clinical Nutrition. Vol.20 (2001), p.41.

[5] S. L. Gorbach: The American Journal of Gastroenterology. Vol.95 (2000), p.2.

[6] H.M. Probert, G.R. Gibson and K.M. Tuohy: Drug Discovery Today. Vol.8(2003), p.692.

[7] K. Hatakka and E. Savilahti et al: British Medical Journal. Vol.322(2001), p.1327.

[8] A.H. Burton and J.E. Tate et al: The Lancet Infectious Diseases. Vol.12(2012), p.136.

[9] J. Nordgren and L.W. Nitiema et al: International Journal of Infectious Diseases. Vol.15(2011), p.646.

[10] D. Sur, B. Manna and S.K. Niyogi et al: Epidemiology\&Infection. Vol.139(2011), p.919.

[11] B.C. Johnston and A.L. Supina et a1: The Cochrane Database of Systematic Reviews. Vol.3(2008), p.280.

[12] J. Slavin: Nutrients. Vol.5(2013). p.1417.

[13] K.W. Reisinger et al: Journal of Pediatric Gastroenterology and Nutrition. Vol.59(2014),p.720.

[14] G. Moro and I. Minoli et al: Journal of Pediatric Gastroenterology and Nutrition. Vol.34(2002),p.291.

[15] R.R. Watson and V.R. Preedy: Bioactive foods in promoting health: Probiotics and prebiotics. (Macmillan Publishing Co., USA, 2010).

[16] L.K. Sarao and M. Arora: Critical Reviews in Food Science and Nutrition. Vol.57(2017),p.344. 